\title{
Hydrostatic pressure induced three-dimensional Dirac semimetal in black phosphorus
}

\author{
Peng-Lai Gong, ${ }^{1}$ Da-Yong Liu, ${ }^{1, *}$ Kai-Shuai Yang, ${ }^{1}$ Zi-Ji Xiang, ${ }^{2}$ Xian-Hui Chen, ${ }^{2}$ \\ Zhi Zeng, ${ }^{1,3}$ Shun-Qing Shen, ${ }^{4}$ and Liang-Jian Zou ${ }^{1,3, \dagger}$ \\ ${ }^{1}$ Key Laboratory of Materials Physics, Institute of Solid State Physics, Chinese Academy of Sciences, \\ P.O. Box 1129, Hefei, 230031, China \\ ${ }^{2}$ Department of Physics, University of Science and Technology of China, Hefei, 230026, China \\ ${ }^{3}$ University of Science and Technology of China, Hefei, 230026, China \\ ${ }^{4}$ Department of Physics, The University of Hong Kong, Pokfulam Road, Hong Kong, China \\ (Received 14 September 2015; revised manuscript received 4 May 2016; published 25 May 2016)
}

\begin{abstract}
We present the first-principles studies on the hydrostatic pressure effect of the electronic properties of black phosphorus. We show that the energy bands crossover around the critical pressure $P_{c}=1.23 \mathrm{GPa}$; with increasing pressure, the band reversal occurs at the $Z$ point and evolves into 4 twofold-degenerate Dirac cones around the $Z$ point, suggesting that pressured black phosphorus is a 3D Dirac semimetal. With further increasing pressure the Dirac cones in the $\Gamma-Z$ line move toward the $\Gamma$ point and evolve into two hole-type Fermi pockets, and those in the $Z-M$ lines move toward the $M$ point and evolve into two tiny electron-type Fermi pockets, and a band above the $Z-M$ line sinks below $\mathrm{E}_{F}$ and contributes four electron-type pockets. A clear Lifshitz transition occurs at $P_{c}$ from semiconductor to 3D Dirac semimetal. Such a 3D Dirac semimetal is protected by the nonsymmorphic space symmetry of bulk black phosphorus. These suggest the bright perspective of black phosphorus for optoelectronic and electronic devices due to its easy modulation by pressure.
\end{abstract}

DOI: 10.1103/PhysRevB.93.195434

\section{MOTIVATIONS}

The van der Waals force between layers makes layered compounds graphite, $\mathrm{MoS}_{2}, \mathrm{WTe}_{2}$ and $\mathrm{TaS}_{2}$, etc., be easily exfoliated to monolayers as two-dimensional materials, such as graphite to graphene and $\mathrm{MoS}_{2}$ to its monolayer. As a possible candidate of optoelectronic and electronic material, the energy gap of black phosphorus varies from $0.3 \mathrm{eV}$ in bulk to about $2 \mathrm{eV}$ in monolayer, filling the optical interval between small energy gap $0-0.3 \mathrm{eV}$ in graphene and large energy gap of $1-2 \mathrm{eV}$ in semiconductive dichalcogenides [1]. Recently, black phosphorus in bulk multilayers and monolayers have received considerable interest [2-4]. Zhang et al. demonstrated that multilayer black phosphorus exhibits as high as $10^{5}$ drain current modulation and $10^{3} \mathrm{~cm}^{2} \mathrm{~V}^{-1} \mathrm{~s}^{-1}$ charge mobility [5], showing that black phosphorus thin film might be a good potential candidate for field effect transistor [6]. More recently it is shown that a moderate hydrostatic pressure could not only drive semiconductive black phosphorus to metallic, but also tune multiple Fermi surfaces and Lifshitz point in the magnetotransport and Shubnikov-De Haas oscillation measurements [7]. These properties make black phosphorus a new exciting field both in material sciences and condensed matter physics [8].

Hydrostatic pressure could easily change the crystal structure of black phosphorus and modify its electronic properties [3,9]. As the most stable allotrope of phosphorus, black phosphorus exhibits three different phases under moderate high hydrostatic pressures: the orthorhombic phase with wrinkled hexagons, the graphene-like rhombohedral phase with hexagonal lattice for the pressure $P>4.5 \mathrm{GPa}$ [10],

\footnotetext{
*dyliu@ theory.issp.ac.cn

${ }^{\dagger}$ zou@theory.issp.ac.cn
}

and the simple cubic phase for $P>10.3 \mathrm{GPa}$ [10]. Under high pressure the P-P bond lengths and bond angle of black phosphorus display strong pressure dependence, and exhibit highly anisotropy in compressibility [9]. Though a strain may crucially modify the energy gap of monolayer black phosphorus [3], it is completely different from the hydrostatic pressure effect on bulk black phosphorus, since the van der Waals force leads to considerable interlayer coupling [11]. One anticipates that a high hydrostatic pressure significantly compresses the interlayer distance of $\mathrm{P}$ atoms, however, it remains a puzzle and unclear scenario: How do the electronic structures evolve with increasing hydrostatic pressures? Especially, how does the Lifshitz point occur under pressure?

On the other hand, searching for 3D Dirac semimetals has attracted great attention in recent years [12,13]. Recently Kim et al. claimed [14] that they observed the Dirac semimetal state by depositing $\mathrm{K}$ ions on the surface of black phosphorus, however, their Dirac cones near the $\Gamma$ point actually always sink below $\mathrm{E}_{F}$. Cava et al. [13] pointed out that a crystal with glide planes and scream axes might form a 3D Dirac semimetal when the electronic structures of the crystal possess a twofolddegenerate band, since the nonsymmorphic symmetry in the crystal lattice leads to the band inversion at a point in the Brillouin zone $[15,16]$. The space group of bulk black phosphorus has a glide plane, thus it is a potential candidate of a 3D Dirac semimetal. To elucidate electronic and optical properties of black phosphorus under hydrostatic pressure, in this paper, using the first-principles electronic structures calculations and analytic studies, we for the first time present the evolutions of the band structures and Fermi surfaces of black phosphorus under hydrostatic pressure, and clearly demonstrate the Lifshitz transition from a semiconductor to a Dirac semimetal. We argue that the nonsymmorphic symmetry in crystal structure of black phosphorus protects it as a 3D Dirac semimetal. 


\section{COMPUTATIONAL STRUCTURES AND PHONON SPECTRA}

\section{A. Computational details}

To explore the evolutions of crystal structures, electronic structures, and physical properties with hydrostatic pressures, we first achieve the stable crystal structures of black phosphorus under various pressures within the framework of density functional theory (DFT) using the projector augmented wave (PAW) method [17,18] as implemented in the Vienna Ab initio Simulation Package (VASP) code [19]. These structures are optimized by using the optB88-vdW method [20,21], so as to take into account the interlayer van der Waals force in bulk black phosphorus.

The experimental lattice parameters of bulk black phosphorus are taken as the initial structure. In our work, the directions of lattice constants $a$ and $b$ are set to be along the zigzag (the $y$ axis) and the armchair (the $x$ axis) directions, respectively, in the crystal structure of bulk black phosphorus; and $c$ is along the interlayer direction (the $z$ axis), as displayed in Fig. 1. Both the shape and volume of each supercell have been relaxed fully at a target pressure and all atoms in the supercell were allowed to move until the residual force per atom was dropped below $0.001 \mathrm{eV} / \AA$. The energy cutoff for the plane-wave basis was set to $500 \mathrm{eV}$ for all calculations. A $k$ mesh of $10 \times 4 \times 8$ in band structure calculation was adopted to sample the first Brillouin zone of the primitive unit cell of bulk black phosphorus. Uniform $k$ mesh of $30 \times 30 \times 30$ was adopted to obtain Fermi surfaces when black phosphorus changes into semimetal. Phonon calculations were carried out using the finite displacement method and PHONONPY package [22,23]. A $3 \times 3 \times 4$ supercell and a $5 \times 5 \times 5 k$ mesh were applied to calculate the force constants.

\section{B. Crystal structure evolution and phonon spectra}

We find that the calculated lattice constants of bulk black phosphorus in the orthorhombic phase under various pressures shown in Fig. 2 are in good agreement with the experimental results $[9,24,25]$. In detail, the calculated lattice constants $a$ and $b$ are very close to the experimental results $[9,24,25]$, though the lattice constant $c$ is slightly larger than the measured within $0.2 \%$, since the GGA functionals with the $v d W$ correction overestimates the layer distance $c$, implying that our theoretical structural parameters are highly reliable. Furthermore, the phonon spectra for our theoretical structures also demonstrate

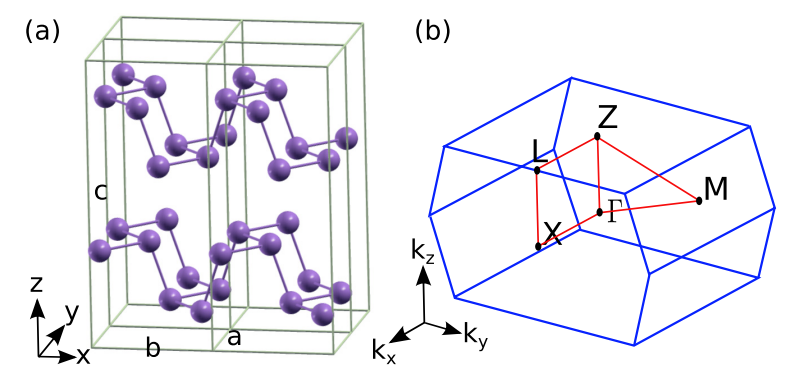

FIG. 1. Crystal structure of bulk black phosphorus (a) and the first Brillouin zone and some high symmetric points of bulk black phosphorus (b).

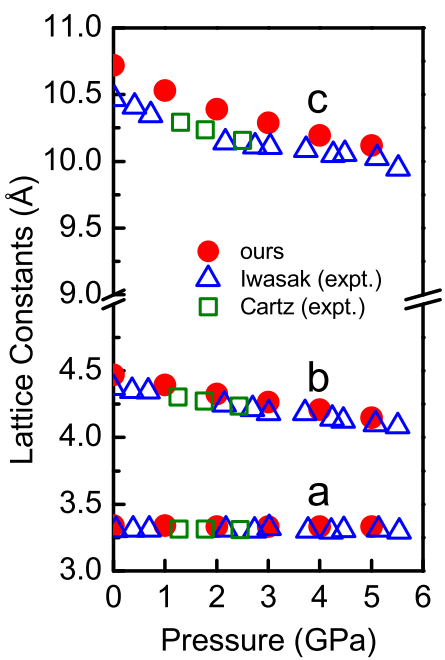

FIG. 2. Lattice constants of bulk black phosphorus under various pressures. The experimental data from Iwasaki et al. [24] and Cartz et al. [9,25] are also shown as open triangles and squares for comparison.

that the pressured structures are stable. Our numerical results have shown that all of our theoretical structures have not any imaginary frequency, as shown in Fig. 10 in the Appendix.

\section{ELECTRONIC STRUCTURES EVOLUTION UNDER PRESSURE}

Electronic band structures were obtained by the VASP method based on the different pressurized structures. All of the calculations are cross-checked by using the QUANTUM ESPRESSO [26] and WIEN2K [27] codes, respectively, and the results are consistent with each other. Within the hybrid functional of Heyd, Scuseria, and Ernzerhof (HSE) framework [28,29], we obtain an energy gap of $0.34 \mathrm{eV}$ at ambient condition, in agreement with the experimental and recent theoretical data $[5,11]$.

To save the computation resource and accord with the experiments [7], throughout this paper we present the numerical results within the modified Becke-Johnson (mBJ) exchange-correlation potential $[30,31]$ for the semiconducting phase, and the PBE exchange-correlation potential [32] for the metallic phase. It is now recognized that similar to the HSE or GW methods, the $\mathrm{mBJ}$ method could well describe a wide range of materials properties, including the band gap of semiconductors, while it sometimes makes false predictions of fundamental properties of metals [33]. During the application of hydrostatic pressure, the black phosphorus undergoes a change from a semiconductive phase to a semimetallic one. It is desirable and self-consistent to adopt the same first-principles method with the same exchange-correlation potential to calculate the electronic properties of black phosphorus over a wide pressure range. However, due to the limit of each method, we have to adopt a different exchange-correlation potential to calculate the energy band of the semiconductor and semimetal.

The pressure dependence of band structures for the semiconductive (mBJ results) and for the semimetallic (PBE result) 

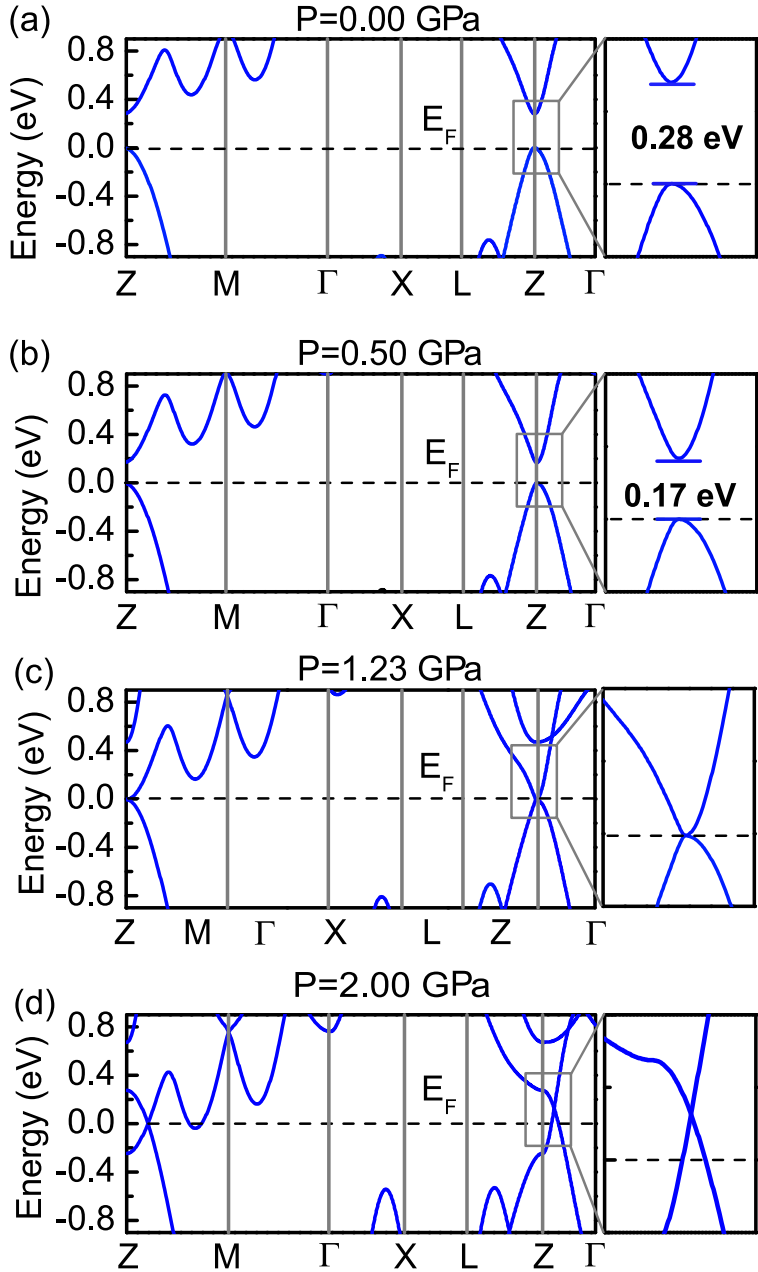

FIG. 3. Evolution of energy band structures of bulk black phosphorus with increasing pressures from $0 \mathrm{GPa}$ (a), $0.5 \mathrm{GPa}$ (b), $1.23 \mathrm{GPa}$, the critical pressure for band crossover (c), 2.0 GPa (d). The first three figures and the last one are the $\mathrm{mBJ}$ and PBE results, respectively.

phases of bulk black phosphorus is shown in Fig. 3. It shows that the system opens a gap about $0.28 \mathrm{eV}$ at $0 \mathrm{GPa}$. With increasing the pressure to 0.5 and $1.0 \mathrm{GPa}$, up to about $P_{c}=1.23 \mathrm{GPa}$, the band gap gradually closes and the two bands below and above $\mathrm{E}_{F}$ touch at the $Z$ point. Thus the critical pressure for the semiconductor-semimetal transition is about $P_{c}=1.23 \mathrm{GPa}$ in the $\mathrm{mBJ}$ functional, which is comparable with the value observed in the recent experiment [7]. Since the energy bands begin to anti-inverse at $P_{c}$, the black phosphorus becomes a semimetal with a degenerate Dirac point at the $Z$ point, as seen the band structures in Fig. 3(c).

When the hydrostatic pressure increases up to $1.5 \mathrm{GPa}$ (not shown), the energy bands already cross with each other around the Fermi energy $\mathrm{E}_{F}$, the anti-inversed energy bands develop the linear dispersions and Dirac points in the $Z-M$ and $Z-\Gamma$ directions, respectively. We attribute to that $p_{z}$ orbitals along the $c$ axis gradually overlap with increasing the pressure, as shown in Fig. 4, majorly contributing to the band anti-inversion. At a pressure of 2.0 GPa shown in Fig. 3(d), the (a)

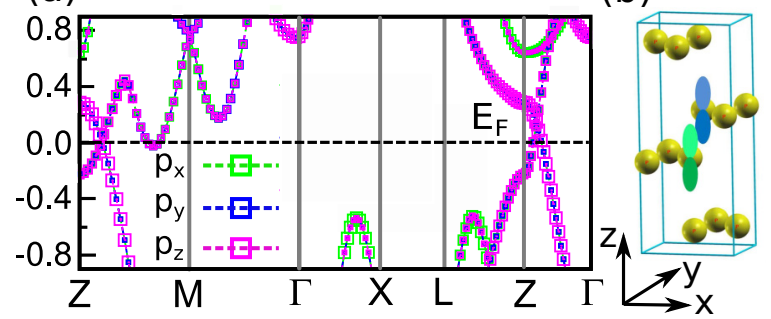

FIG. 4. Bands structure with orbital projection of bulk black phosphorus at $2.0 \mathrm{GPa}$ (a) and schematic diagram of $3 p_{z}$ orbital distribution contributed to the Dirac cones (b).

Dirac cones in the $Z-\Gamma$ direction shift toward the $\Gamma$ point and develops two hole Fermi pockets. On the other hand, the Dirac cones in the $Z-M$ line shift toward the $M$ point and above $\mathrm{E}_{F}$ in energy, contributing two tiny electron pockets; meanwhile a band above the $Z-M$ line sinks below $\mathrm{E}_{F}$ and develops four electron Fermi pockets. At pressures up to $2.5 \sim 4 \mathrm{GPa}$ we studied, the volumes of the hole Fermi pocket gradually expand, and an electron Fermi pocket in the $Z-M$ line becomes considerably large, in agreement with recent experimental observation [7]. Thus it is naturally expected that the hole and electron carriers should coexist under high pressure until 4 $\mathrm{GPa}$, since high hydrostatic pressure does not break the charge balance.

As well known, the wide bandwidth $2 p_{z}$ orbitals of carbon atoms contributes the six Dirac cones of graphene. One may wonder which orbital or which a few orbitals contribute the Dirac cones when the hydrostatic pressure $P>P_{c}$. After projecting the weight of orbitals, we could find that the $3 p_{z}$ orbitals contribute the dominant weight of the Dirac cones. As seen in Fig. 4, under the high hydrostatic pressure, the $3 p_{z}$ orbitals overlap with each other, and lead to the linearized spectrum.

\section{DIRAC CONES AND 3D DIRAC SEMIMETAL}

From the band structures one finds that when the hydrostatic pressure increases to the critical value of $P_{c}=1.23 \mathrm{GPa}$, the valence band and the conduction band touch together at the high-symmetric $Z$ point of the Brillouin zone and gradually intersect with each other. The energy band structures of the system exhibit anti-inversion under further pressure. As described above, when the pressure becomes larger than the critical point, $P>P_{c}$, the anti-inverted points at the high-symmetric $Z$ point evolve into Dirac points around the $Z$ point, and this fourfold-degenerate point gradually splits into four Dirac cones with the increase of pressure, as sketched in Fig. 5 and confirmed by Fermi surfaces evolution under pressures shown later. Taking into account the spin degree of freedom, each Dirac cone remains twofold degenerate. Note that these Dirac cones are twofold degenerate once taking into account the spin freedom of degree, thus the influence of magnetic field deserves further investigation.

The 3D Dirac semimetal can further be verified from the density of states (DOS) of black phosphorus near the critical pressure $P_{c}$ shown in Fig. 6. One observes that the 


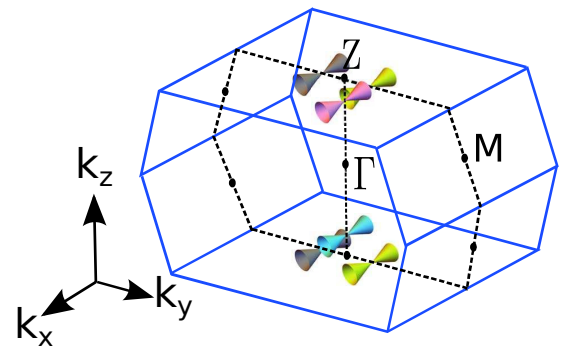

FIG. 5. Sketched distribution of four Dirac cones in the first Brillouin zone of bulk black phosphorus under the pressure of 2.0 $\mathrm{GPa}$. The two Dirac cones lie on the upper and lower surfaces of the Brillouin zone are identical.

DOS vanishes near $\mathrm{E}_{F}$, suggesting well-defined semimetallic nature. These properties strongly indicate that bulk black phosphorus under intermediate pressure is the first realistic example of pressure-modified 3D Dirac semimetal. At $P=$ 3.0 GPa shown in Fig. 6, finite DOS appears near $\mathrm{E}_{F}$ due to the upward and downward bands, implying that the system becomes metallic. However, the DOS near $\mathrm{E}_{F}$ does not increase considerably.

The appearance of the band inversion, Dirac points, and Dirac cones in black phosphorus is particularly interesting, since in this calculation, neither considerable spin-orbit coupling is taken into account, nor a graphenelike honeycomb structure is realized in black phosphorus, though the crystal structure of black phosphorus changes significantly. This raises a question: What is the physical origin of the formation of the Dirac semimetal? Considering the space-group symmetry operators of the black phosphorus, we attribute the origin of Dirac points to the symmetry of the nonsymmorphical space group in black phosphorus. In the hydrostatic pressure range we studied, the space group of the crystal structure of black phosphorus is always Cmca, which possesses a nonsymmorphical operation of a glide along the $c$ axis. Under these symmetrical operations $\hat{U}$, the Hamiltonian of the black phosphorus, $\mathrm{H}(\mathrm{k})$, satisfies:

$$
H(\mathbf{k})=U^{*}(k) H(\hat{U} \mathbf{k}) U(\mathbf{k}) .
$$

At $k_{z}=\pi / c$, or the Brillouin zone surface containing the $Z$ point $(0,0, \pi / c)$, the energy band structures of a nonsymmorphical crystal may be connected, leading to the

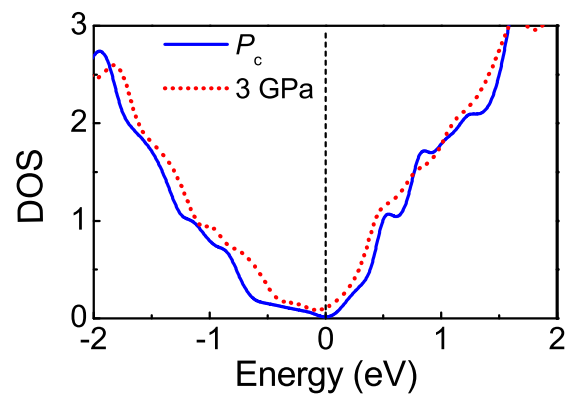

FIG. 6. Density of states (DOS) of bulk black phosphorus under the pressure of $P_{c}=1.23 \mathrm{GPa}$ (blue solid line) and 3.00 GPa (red dashed line).

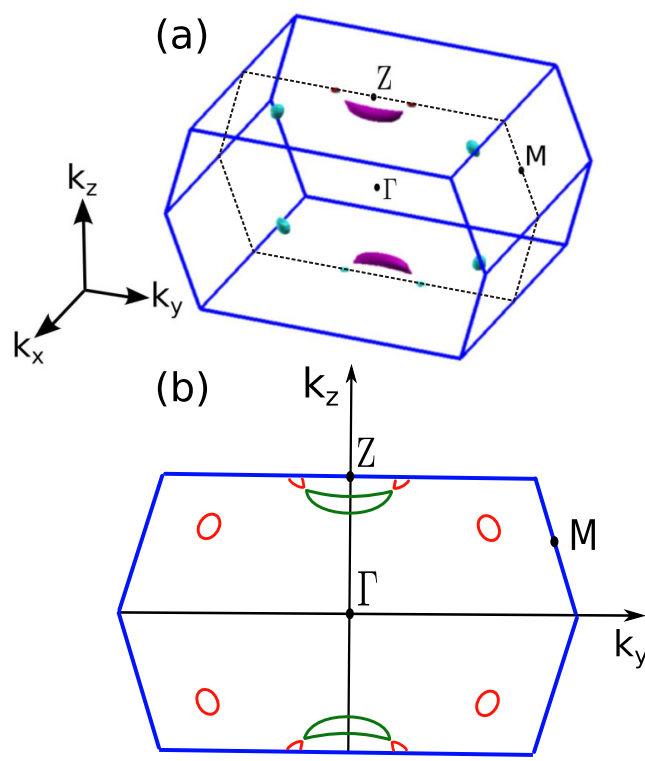

FIG. 7. Fermi surface (a) and its projection (b) of bulk black phosphorus under the pressure of $2.0 \mathrm{GPa}$. The projection on the plane $\left(0, k_{y}, k_{z}\right)$ is shown. The two pockets in the $\Gamma-Z$ line are hole type, and the others are electron type.

degeneracy [34]. Such a connection and degeneracy lead to the fourfold-degenerate Dirac points $[13,35]$.

\section{FERMI SURFACES EVOLUTION WITH PRESSURES}

From the preceding studies we have shown that critical pressure $P_{c}$ is a Lifshitz point, which the topology of Fermi surface of bulk black phosphorus changes crucially. The Fermi surfaces and its cross section of black phosphorus under the pressure of $2.0 \mathrm{GPa}$ are displayed in Fig. 7. There are three different kinds of Fermi pockets: two hole pockets arising from the upward shift of two Dirac cones in the $\Gamma-Z$ line above $\mathrm{E}_{F}$, and two tiny electron pockets on the upper and lower surface of the Brillouin zone arising from the sink of two Dirac cones in the $M-Z$ line below $\mathrm{E}_{F}$; another four electron-type pockets in

TABLE I. Extremal cross-section area $S_{F}$ of electron and hole pockets at pressure 2.0 GPa. $\beta, \alpha$, and $\alpha^{*}$ stand for a Dirac dispersion hole pocket at $Z-\Gamma$ line, a normal electron pocket and a Dirac dispersion electron pocket at $Z-M$ line, respectively.

\begin{tabular}{lccc}
\hline \hline Pockets type & $\beta$ (hole) & $\alpha$ (electron) & $\alpha^{*}$ (electron) \\
\hline$S_{F}\left(\mathrm{~nm}^{-2}\right)$ & $1.06^{\mathrm{a}}$ & $0.4^{\mathrm{a}}$ & $0.23^{\mathrm{a}}$ \\
& $(0.42)^{\mathrm{b}}$ & $(0.22)^{\mathrm{b}}$ & $(0.16)^{\mathrm{c}}$ \\
Inequivalent numbers & 2 & 4 & 2 \\
Total $S_{F}\left(\mathrm{~nm}^{-2}\right)$ & 2.12 & 1.60 & 0.46 \\
\hline
\end{tabular}

${ }^{\mathrm{a} O u r s,}$ the plane $\left(0, k_{y}, k_{z}\right)$.

${ }^{\mathrm{b}}$ Ref. [7], the plane $\left(k_{x}, k_{y}, 0\right)$, with the magnetic field $H=20 \mathrm{~T}$ parallelled to the $c$ axis and under the pressure of $2.0 \mathrm{GPa}$.

${ }^{\mathrm{c}}$ Ref. [7], the plane $\left(k_{x}^{\prime}, k_{y}, k_{z}\right)$, where the direction of $k_{x}^{\prime}$ is $18^{\circ}$ away from the $k_{x}$ direction, with $H=20 \mathrm{~T}$ and under the pressure of $2.4 \mathrm{GPa}$. 


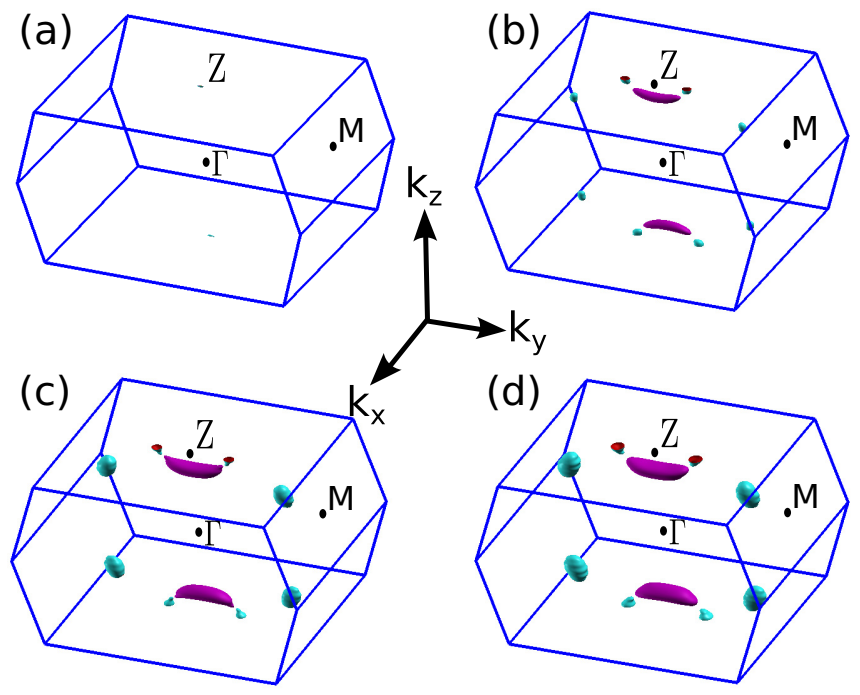

FIG. 8. Calculated Fermi surfaces for bulk black phosphorus from 1.23 $\mathrm{GPa}(\mathrm{a}), 1.5 \mathrm{GPa}(\mathrm{b}), 3.0 \mathrm{GPa}(\mathrm{c})$, and 4.0 $\mathrm{GPa}(\mathrm{d})$.

the $Z-M$ line come from the sink of the bands below $\mathrm{E}_{F}$. These four Fermi pockets do not originate from the Dirac cones.

The cross-section Fermi surface on the plane $\left(0, k_{y}, k_{z}\right)$ at 2.0 GPa is displayed in Fig. 7(b). This shows that the Dirac points and the centers of the Fermi pockets are on the $k_{x}=0$ plane. The extremal cross-section area $S_{F}$ of the electron and hole pockets in the plane $\left(0, k_{y}, k_{z}\right)$ are estimated. Our results show $S_{F}$ are $0.40,0.23$ and $1.06 \mathrm{~nm}^{-2}$ for a large electron pocket $(\alpha)$, a tiny electron pocket $\left(\alpha^{*}\right)$ and a hole pocket $(\beta)$, respectively, listed in Table I. As for Shubnikov-De Haas oscillation experiment [7], it reports that 0.22 for $\alpha$ and $0.42 \mathrm{~nm}^{-2}$ for $\beta$ in the plane $\left(k_{x}, k_{y}, 0\right)$, with the magnetic field $H=20 \mathrm{~T}$ parallelled to the $c$ axis and under the pressure of $2.0 \mathrm{GPa}$; while $S_{F}$ for $\alpha^{*}$ is $0.16 \mathrm{~nm}^{-2}$ in $\left(k_{x}^{\prime}, k_{y}, k_{z}\right)$ with $H=20 \mathrm{~T}$ and under the pressure of $2.4 \mathrm{GPa}$. Our theoretical results have the same order magnitude with the experiment [7]. With consideration of the number of two types of pockets ( 2 for $\beta, 4$ for $\alpha$ and 2 for $\alpha^{*}$ ) in the first Brillouin zone, the total $S_{F}$ S of electron pockets and hole pockets are approximately equal to $2.00 \mathrm{~nm}^{-2}$. The numbers of two kinds of carriers keep identical, indicating a complete compensation. These theoretical data show that the system is semimetal state, in good agreement with the results by the field dependence of transverse magnetoresistance experiment [7]. Also, the Dirac fermions and normal fermions coexist in the black phosphorus under the pressure larger than $P_{c}$, providing a complete novel quantum system.
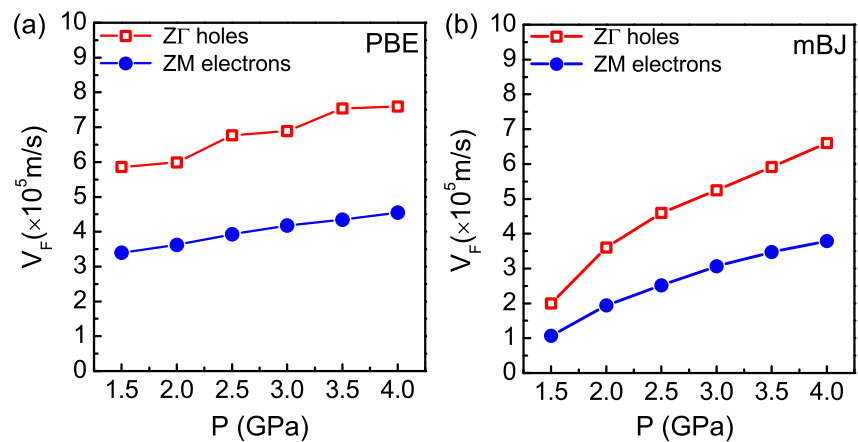

FIG. 9. Dependence of the average Fermi velocity of bulk black phosphorus under the pressures from 1.5 GPa up to $4.0 \mathrm{GPa}$ by the PBE (a) and the $\mathrm{mBJ}$ potentials (b), respectively.

The pressures can modify the electronic structures, leading to the interesting evolution of Fermi surfaces, and driving significant change in the topology of electronic structures, i.e., the Lifshitz transition. We present Fermi surfaces topology of bulk black phosphorus under different pressures in Fig. 8. It is shown that Fermi surface is null before the critical pressure for the semiconductor-semimetal transition. With increasing the pressure to 1.5 and $3.0 \mathrm{GPa}$, up to $4.0 \mathrm{GPa}$, the volumes of hole Fermi pockets in $\Gamma-Z$ line gradually expand, and electron Fermi pockets in $Z-M$ line grow largely, consistent with the resent experimental results [7]. The volumes of electron and hole pockets become large with increasing pressure, indicating that more and more hole and electron carriers are produced in the system.

\section{ANISOTROPIC PHYSICAL PROPERTIES}

The anisotropic electronic structures in black phosphorus result in highly orientation-dependent properties, such as the effective masses of carriers and Fermi velocities, etc. It displays the hydrostatic pressure dependence of the Fermi velocities $\left(V_{F}\right)$ of the hole and electron carriers by the PBE potential shown in Fig. 9(a) and the mBJ one in Fig. 9(b), respectively. We focus on the Fermi velocity of Dirac cones near the Fermi level, which is important for the fundamental properties of the system. Note that we calculate the average Fermi velocity from two branches of Dirac cone. It has shown that the considerable pressure dependence of the Fermi velocity both for the PBE and the mBJ results, while, the pressure dependence of $\mathrm{V}_{F}$ by the $\mathrm{mBJ}$ potential is significantly larger than that by the PBE potential. Of course,

TABLE II. Effective masses of black phosphorus under various hydrostatic pressures and comparison with Refs. [11,37].

\begin{tabular}{|c|c|c|c|c|}
\hline & $\mathrm{P}(\mathrm{GPa})$ & $m_{x}^{*} / m_{0}$ & $m_{y}^{*} / m_{0}$ & $m_{z}^{*} / m_{0}$ \\
\hline \multirow{3}{*}{ e } & 0.00 & $0.14(0.12[11], 0.08[37])$ & $1.26(1.15[11], 1.03[37])$ & $0.16(0.15[11], 0.13[37])$ \\
\hline & 0.50 & 0.09 & 1.24 & 0.14 \\
\hline & 1.00 & 0.05 & 1.21 & 0.13 \\
\hline \multirow{3}{*}{$\mathrm{h}$} & 0.00 & $0.12(0.11[11], 0.07[37])$ & $0.90(0.71[11], 0.65[37])$ & $0.34(0.30[11], 0.28$ [37]) \\
\hline & 0.50 & 0.08 & 0.82 & 0.32 \\
\hline & 1.00 & 0.04 & 0.78 & 0.31 \\
\hline
\end{tabular}



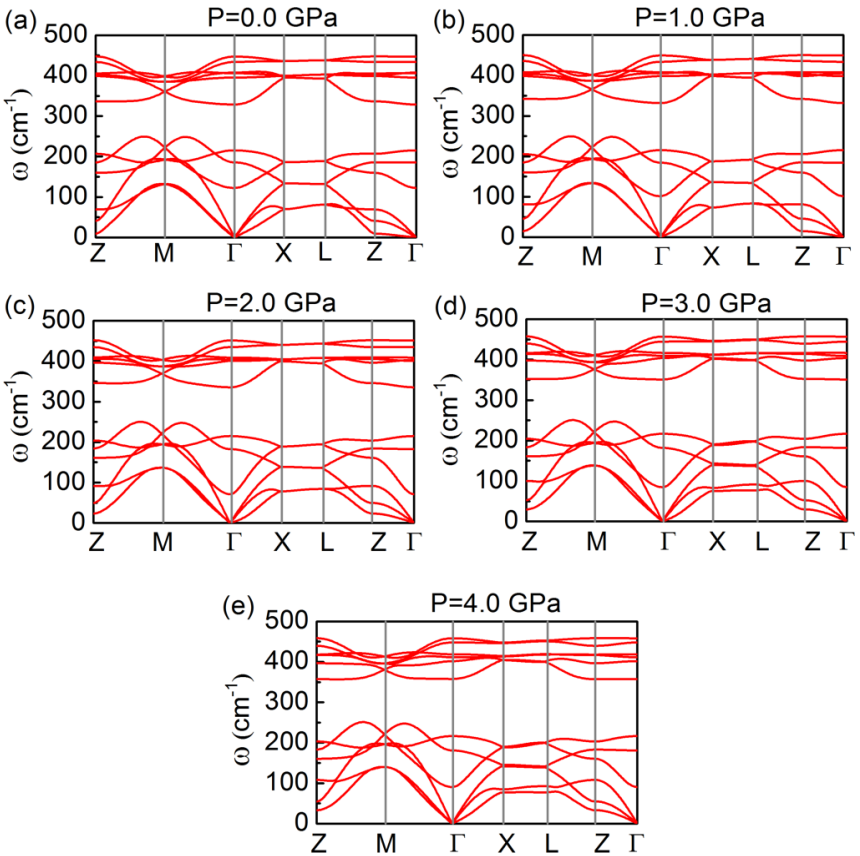

FIG. 10. Calculated phonon dispersion of bulk black phosphorus under pressures of $P=0,1,2,3$, and $4 \mathrm{GPa}$.

more elaborated experiments are expected to confirm our theoretical details in the future.

In addition, it is seen that the Fermi velocities monotonically increase with increasing the hydrostatic pressure. The velocities along the $Z-\Gamma$ direction are almost twice of those along the $Z-M$ direction, showing strong transport anisotropy. Meanwhile, the effective masses of the hole and electron carriers also display considerable anisotropy. As listed in Table II, with increasing hydrostatic pressure, the effective masses of electron carriers in the $y$ and $z$ axis almost do not alter, and the effective masses of hole carriers along the $z$ axis change slightly. However, the effective masses along the $x$ axis decrease to about one third of the ambient pressure, demonstrating strong pressure modulation.

\section{SUMMARY}

This paper presents the nontrivial influence of hydrostatic pressure on bulk black phosphorus, which is distinctly different from the strain-induced effect [3]. The hydrostatic pressure shortens the distance of two $\mathrm{P}$ atomic layers and the height of $\mathrm{P}$ atomic layers, which results in considerable changes in electronic structures and Fermi surface topology, hence a Lifshitz transition. It also leads to the appearance of four twofold-degenerate Dirac cones at a critical pressure $P_{c}$, implying that black phosphorus under hydrostatic pressure becomes a 3D Dirac semimetal. More recent work suggested that these Dirac points may form unusual nodal rings in pressured black phosphorus [36] These show that pressure controlled layered black phosphorus may have potential application in optoelectronic and electronic devices.

\section{ACKNOWLEDGMENTS}

The authors L.-J.Z. and D.-Y.L. thank X.-G. Gong for his hospitality during their visit to the Department of Physics, Fudan University. This work was supported by the NSF of China under Grant Nos. 11474287, 11274310, 11574315 and the key project 11534010 . Numerical calculations were performed at the Center for Computational Science of CASHIPS.

\section{APPENDIX}

To make sure that pressured structures are thermodynamically stable, we calculate the phonon spectra of bulk black phosphorus for our theoretical structures under the hydrostatic pressures from $0 \mathrm{GPa}$ to $4 \mathrm{GPa}$, as shown in Fig. 10. Our results show that the phonon spectra of these structures have no any imaginary frequency, implying that the optimized geometries really locate at the minimum point of the potential surface.
[1] F. Xia, H. Wang, D. Xiao, M. Dubey, and A. Ramasubramaniam, Nat. Photonics 8, 899 (2014).

[2] T. Low, R. Roldán, H. Wang, F. Xia, P. Avouris, L. M. Moreno, and F. Guinea, Phys. Rev. Lett. 113, 106802 (2014).

[3] A. S. Rodin, A. Carvalho, and A. H. Castro Neto, Phys. Rev. Lett. 112, 176801 (2014).

[4] V. Tran, R. Soklaski, Y. Liang, and L. Yang, Phys. Rev. B 89, 235319 (2014).

[5] L. K. Li, Y. J. Yu, G. J. Ye, Q. Q. Ge, X. D. Ou, H. Wu, D. L. Feng, X. H. Chen, and Y. B. Zhang, Nature Nanotech. 9, 372 (2014).

[6] F. Xia, H. Wang, and Y. Jia, Nature Commun. 5, 4458 (2014).

[7] Z. J. Xiang, G. J. Ye, C. Shang, B. Lei, N. Z. Wang, K. S. Yang, D. Y. Liu, F. B. Meng, X. G. Luo, L. J. Zou, Z. Sun, Y. B. Zhang, and X. H. Chen, Phys. Rev. Lett. 115, 186403 (2015).

[8] Y. Du, H. Liu, Y. Deng, and P. D. Ye, Acs Nano 8, 10035 (2014).
[9] L. Cartz, S. R. Srinivasa, R. J. Riedner, J. D. Jorgensen, and T. G. Worlton, J. Chem. Phys. 71, 1718 (1979).

[10] M. Okajima, S. Endo, Y. Akahama, and S.-I. Narita, Jpn. J. Appl. Phys. 23, 15 (1984).

[11] J. Qiao, X. Kong, Z.-X. Hu, F. Yang, and W. Ji, Nature Commun. 5, 4475 (2014).

[12] Z. Wang, Y. Sun, X.-Q. Chen, C. Franchini, G. Xu, H. Weng, X. Dai, and Z. Fang, Phys. Rev. B 85, 195320 (2012).

[13] Q. D. Gibson, L. M. Schoop, L. Muechler, L. S. Xie, M. Hirschberger, N. P. Ong, R. Car, and R. J. Cava, Phys. Rev. B 91, 205128 (2015).

[14] J. Kim, S. S. Baik, S. H. Ryu, Y. Sohn, S. Park, B.-G. Park, J. Denlinger, Y. Yi, H. J. Choi, and K. S. Kim, Science 349, 723 (2015).

[15] L. Michel and J. Zak, Phys. Rev. B 59, 5998 (1999).

[16] J. Zak, J. Phys. A: Math. Gen. 35, 6509 (2002). 
[17] P. E. Blöchl, Phys. Rev. B 50, 17953 (1994).

[18] G. Kresse and D. Joubert, Phys. Rev. B 59, 1758 (1999).

[19] G. Kresse and J. Furthmüller, Phys. Rev. B 54, 11169 (1996).

[20] J. Klimeš, D. R. Bowler, and A. Michaelides, Phys. Rev. B 83, 195131 (2011).

[21] J. Klimeš, D. R. Bowler, and A. Michaelides, J. Phys.: Condens. Matter 22, 022201 (2010).

[22] A. Togo, F. Oba, and I. Tanaka, Phys. Rev. B 78, 134106 (2008).

[23] A. Togo, Phonopy, http://phonopy.sourceforge.net/.

[24] T. Kikegawa and H. Iwasaki, Acta Crystallogr., B 39, 158 (1983).

[25] A. Brown and S. Rundqvist, Acta Crystallogr., 19, 684 (1965).

[26] P. Giannozzi, S. Baroni, N. Bonini, M. Calandra, R. Car, C. Cavazzoni, D. Ceresoli, G. L. Chiarotti, M. Cococcioni, and I. Dabo, J. Phys.: Condens. Matt. 21, 395502 (2009).

[27] P. Blaha, K. Schwarz, G. Madsen, D. Kvasnicka, and J. Luitz, An Augmented Plane Wave and Local Orbitals Program for Calculating Crystal Properties (Technical University of Wien, Vienna, 2001).
[28] J. Heyd, G. E. Scuseria, and M. Ernzerhof, J. Chem. Phys. 118, 8207 (2003).

[29] J. Heyd, G. E. Scuseria, and M. Ernzerhof, J. Chem. Phys. 124, 219906 (2006).

[30] A. D. Becke and E. R. Johnson, J. Chem. Phys. 124, 221101 (2006).

[31] F. Tran and P. Blaha, Phys. Rev. Lett. 102, 226401 (2009).

[32] J. P. Perdew, K. Burke, and M. Ernzerhof, Phys. Rev. Lett. 77, 3865 (1996).

[33] W. Gao, T. A. Abtew, T. Cai, Y. Y. Sun, S. B. Zhang, and P. Zhang, arXiv:1504.06259.

[34] G. Lee, J. Kim, and J. Zak, J. Phys.: Condens. Matter 15, 2005 (2003).

[35] H. Weng, X. Dai, and Z. Fang, Phys. Rev. X 4, 011002 (2014).

[36] J. Z. Zhao, R. Yu, H. M. Weng, and Z. Fang, arXiv:1511.05704.

[37] A. Morita and T. Sasaki, J. Phys. Soc. Jpn. 58, 1694 (1989). 